\section{Prevalence, phenotype and genotype of oral enterococci}

Sedgley CM, Lennan SL, Clewell DB. Prevalence, phenotype and genotype of oral enterococci.

Oral Microbiol Immunol 2004: 19: 95-101. (C) Blackwell Munksgaard, 2004

This study investigated the prevalence, phenotype and genotype of oral enterococci. Enterococci were detected in oral rinse samples from $11 \%$ of 100 patients receiving endodontic treatment and $1 \%$ of 100 dental students with no history of endodontic treatment $(P=0.0027)$. All enterococcal isolates were identified as Enterococcus faecalis. Viable counts ranged from $1 \times 10$ to $6 \times 10^{3}$ colony forming units per $\mathrm{mL}$ of oral rinse sample. Potential virulence traits expressed by oral E. faecalis strains included production of hemolysin $(n=4)$ and gelatinase $(n=4)$, and response to pheromones in E. faecalis culture filtrate $(n=1)$. Six strains produced bacteriocin. All strains were susceptible to ampicillin, benzylpenicillin, gentamicin and vancomycin. There was no evidence of metal-ion resistance. One isolate produced hemolysin, gelatinase and bacteriocin, was resistant to several antibiotics, and responded to the pheromone cPD1. Pulsed-field gel electrophoresis and plasmid analysis showed that oral E. faecalis exhibited widespread genetic polymorphism, with plasmids detected in seven strains.

\author{
C. M. Sedgley ${ }^{1}$, S. L. Lennan ${ }^{1}$, \\ D. B. Clewell ${ }^{2}$ \\ Departments of ${ }^{1}$ Cariology, Restorative \\ Sciences and Endodontics, ${ }^{2}$ Biologic and \\ Material Sciences, University of Michigan \\ School of Dentistry, Ann Arbor, MI, USA
}

Key words: oral enterococci; genotype; phenotype; prevalence

Dr C. M. Sedgley, Department of Cariology, Restorative Sciences and Endodontics, University of Michigan Dental School, 1011 North University Drive, Ann Arbor, MI 48109-1078, USA

E-mail: csedgley@umich.edu

Accepted for publication October 6, 2003
Enterococci are gram-positive cocci that form part of the normal gastrointestinal tract flora in animals and humans (12). Clinical studies have shown that enterococci usually make up a small proportion of the flora in the untreated root canal $(4,27)$. However, enterococci have long been implicated in persistent root canal infections $(3,4$, 11) and identified as the species most commonly recovered from root canals of teeth with failed endodontic treatment $(20,28)$.

It is feasible that the root canal species originate in the oral cavity, but data on oral prevalence of enterococci vary $(14,22)$. Enterococci were detected in samples from multiple oral sites in $60 \%$ of 20 laboratory personnel, $60 \%$ of 30 schoolchildren with high caries activity and $75 \%$ of eight endodontic patients (14). In contrast, subgingival enterococci were recovered from only $1 \%$ of 100 early-onset periodontitis patients and $5.1 \%$ of 545 adult periodontitis patients (22). In view of the limited data, the purpose of this study was to examine the oral prevalence, phenotype and genomic profiles of oral enterococci recovered from endodontic patients and from a cohort of subjects not requiring endodontic treatment.

\section{Materials and methods Study groups}

The two study groups consisted of 100 endodontic patients attending the University of Michigan Graduate Endodontic Clinic and 100 dental students with no history of endodontic treatment enrolled at the University of Michigan. Approval to conduct the study was obtained from the University of Michigan Institutional Review Board. Written consent to participate in the study was obtained from all participants. In addition to obtaining an oral rinse sample, information was collected on age, gender, smoker status, medication and details of endodontic treatment. In cases where enterococci were detected in oral rinse samples from endodontic patients, data on active caries status, overall oral hygiene assessment and number of dental restorations were retrospectively obtained from patient records.

\section{Oral rinse sampling and laboratory procedures}

Oral rinse samples were obtained from all participants. The sampling and processing procedures were based on the concentrated oral rinse method (23). Patients were asked to rinse their mouths for $60 \mathrm{~s}$ with $10 \mathrm{ml}$ sterile distilled water from a $50 \mathrm{ml}$ Falcon polypropylene tube (Becton Dickinson Labware, Franklin Lakes, NJ). Denturewearing subjects did not remove their prostheses. After $60 \mathrm{~s}$ the patients returned the oral rinse to the tube, which was then immediately transferred to the laboratory for processing or stored at $4{ }^{\circ} \mathrm{C}$ before transfer. All samples were processed in the laboratory within $5 \mathrm{~h}$ of sampling. Each sample was centrifuged for $10 \mathrm{~min}$ at 10,000 r.p.m. and the supernatant 
discarded. The pellet was resuspended in $1 \mathrm{ml}$ of sterile distilled water and vortex mixed for $30 \mathrm{~s}$, producing the final concentrated suspension, $50 \mu l$ of which was plated onto each of blood agar [Todd Hewitt Broth (THB, Difco; Becton, Dickinson and Company, Sparks, MD) supplemented with $1.5 \%$ agar and $4 \%$ horse blood (Colorado Serum Co., Denver, CO)] and bile esculin agar (Difco) using a Spiral Plater (Model D, Spiral Systems, Inc. Cincinnati, OH). The spiral plater mechanically dispenses an adjustable volume of the sample onto the agar plate in an Archimedian spiral, enabling quantification of microorganisms in a liquid sample, a technique found to correlate well with conventional methods of quantification (31). Following inoculation, agar plates were incubated aerobically for $24-48 \mathrm{~h}$ at $37^{\circ} \mathrm{C}$. After incubation, all plates were examined, and colony forming units counted on bile esculin agar plates. No attempts were made to obtain quantitative data from blood agar plates that were included to serve as positive controls for the more selective bile esculin agar media.

Colonies presumptively identified as enterococci based on bile esculin hydrolysis were purified. Conventional biochemical reactions were used to further characterize the organisms (13). Those isolates characterized as gram-positive cocci, catalase-negative, non-motile, pyruvate-positive, and capable of growth in THB supplemented with $6.5 \% \mathrm{NaCl}$ at $42^{\circ} \mathrm{C}$ were identified using API 20 Strep identification kits (Analytical Profile Index; Bio Mérieux SA, France), with type strain Enterococcus faecalis ATCC 47077 serving as a positive control. All isolates were identified as E.faecalis.

\section{Hemolysin and gelatinase production}

Oral E. faecalis strains were assessed for hemolytic activity on THB agar supplemented with $4 \%$ horse blood. Samples from single colonies were inoculated onto blood agar plates and incubated at $37^{\circ} \mathrm{C}$ for $24 \mathrm{~h}$. Hemolysin activity was determined as a clear halo around the colonies.

Gelatinase activity was assessed using samples from single colonies inoculated onto agar containing 3\% gelatin and incubated at $37^{\circ} \mathrm{C}$ for $24-48 \mathrm{~h}$. Gelatinase activity was evident as a clear halo around the colonies.

\section{Pheromone response}

Oral E.faecalis strains were assessed for pheromone response using microtiter dilu- tion assays as previously described (9). Culture filtrate pheromones were prepared from culture filtrates of plasmid-free E. faecalis JH2-2. Pheromones cAM373, cCF10, cPD1 and cAD1 were synthesized at the Protein Structure Facility, University of Michigan (Ann Arbor, MI). E. faecalis DS16 pAD1, E. faecalis OG1X pAM373, E. faecalis OG1SS pCF10, E. faecalis 39-5 pPD1 and E.faecalis OG1X pAD1 were used as positive controls for pheromone response (5). Briefly, strains were grown to early stationary phase in THB and diluted 10 -fold in fresh THB. All assays were performed in $8 \times 12$ microtiter trays. Pheromone solution $(100 \mu \mathrm{l}$ of $100 \mathrm{ng} / \mathrm{ml}$ in THB) was placed in the first well of the assigned row and serially diluted (twofold) into $50 \mu \mathrm{l}$ THB. E. faecalis strains $(50 \mu 1$ bacterial suspension grown overnight in THB and diluted 1:10 in THB) were added to each well in the assigned row. Trays were incubated for $4 \mathrm{~h}$ at $37^{\circ} \mathrm{C}$ on an orbital shaker at $200 \mathrm{rpm}$. The pheromone titer was defined as the highest dilution of culture filtrate that induced aggregate formation (clumping) in responder cells.

\section{Bacteriocin production}

Oral E.faecalis strains were tested for production of bacteriocin activity essentially as previously described (18). Positive and negative control bacteriocin producer strains were E.faecalis DS16 and E. faecalis FA2-2, respectively (18). Indicator strains were E.faecalis FA2-2, E. faecalis DS16, Enterococcus faecium 409 and Staphylococcus aureus ATCC 6538 grown in THB, and Escherichia coli $\mathrm{DH} 5 \alpha$; and E. coli ATCC 29417 grown in LB broth (Difco) to stationary phase. An inoculum of $10 \mu \mathrm{l}$ of each indicator strain was added to $3 \mathrm{ml}$ of liquefied soft agar $(0.75 \%)$ and poured on a THB agar plate. When this had solidified, samples from single colonies from appropriate producer strains were stabbed into the agar. After aerobic incubation overnight at $37^{\circ} \mathrm{C}$, clear zones were visible around the stabs of bacteriocinogenic strains.

\section{Antimicrobial susceptibility}

Minimal inhibitory concentrations (MICs) were determined using the E-test (AB Biodisk, Solna, Sweden) (2) following the procedures recommended by the manufacturer. Antibiotics tested were ampicillin, benzylpenicillin, chloramphenicol, clindamycin, erythromycin, fusidic acid, gentamicin, kanamycin, rifampin, strepto- mycin, tetracycline and vancomycin. Bacterial suspensions were inoculated onto Mueller-Hinton agar (Difco). Following application of E-test strips, plates were incubated aerobically at $35^{\circ} \mathrm{C}$ for $16-20 \mathrm{~h}$ and MICs read from the interpretive scale at the point at which the ellipse of inhibition intersected the strip.

\section{Metal-ion susceptibility}

Minimal inhibitory concentrations of silver nitrate $\left(\mathrm{AgNO}_{3}\right)$, cadmium sulfate $\left(\mathrm{CdSO}_{4}\right)$, copper sulfate $\left(\mathrm{CuSO}_{4}\right)$, mercury chloride $\left(\mathrm{HgCl}_{2}\right)$ and zinc sulfate $\left(\mathrm{ZnSO}_{4}\right)$ were determined for oral E. faecalis and compared to laboratory strains E. faecalis FA2-2 and E. faecalis OG1RF in broth micro dilution susceptibility assays using microtiter trays.

\section{Pulsed-field gel electrophoresis (PFGE) and plasmid DNA preparation}

Genetic profiles were obtained using pulsed-field gel electrophoresis (PFGE). DNA isolation and digestion for PFGE was essentially as previously described (8). Small-scale plasmid DNA preparations were obtained using an alkaline lysis method as previously described (33). Ethidium bromide-CsCl gradient-prepared plasmid DNA was isolated as previously described (6). Restriction enzymes and buffers were obtained from Invitrogen and restriction analysis of samples was performed under conditions recommended by the manufacturer. Genomic DNA restriction fragments were separated on $0.8 \%$ agarose gels in TBE buffer. Plasmid DNA restriction fragments were separated on $0.7 \%$ agarose gels in TAE buffer. The gels were stained with ethidium bromide for $30 \mathrm{~min}$ and the bands were visualized by fluorescence under UV light.

\section{Statistical analysis}

The unpaired $t$-test was used to compare the mean ages of participants in each sampling group. Fisher's exact tests were used to compare prevalence of oral E. faecalis, smoking and medication usage in each sampling group and to analyze potential factors associated with oral prevalence of E. faecalis in endodontic patients. Significance was defined as $P<0.05$.

\section{Results \\ Sampling groups}

There were 42 females and 58 males in the endodontic patient group. Reasons 
for attendance were initial endodontic treatment $(n=52)$, mid-treatment $(n=26)$, endodontic retreatment $(n=19)$ and recall $(n=3)$. There were 47 females and 53 males in the dental student group. The mean age of endodontic patients (47.2 years, SD 15.9) was significantly higher than the mean age of dental students (23.9 years, SD 2.2) $(P<0.0001)$. There were significantly more smokers in the patient group $(n=27)$ than in the student group $(n=5)(P<0.0001)$ and significantly more patients $(n=24)$ were taking antibiotics or had taken antibiotics within the past 2 months compared to students $(n=13)(P=0.0478)$. There was no statistically significant difference in the numbers of subjects taking medication that had the potential to reduce salivary flow in the patient group $(n=12)$ compared to the student group $(n=8)$.

\section{Oral prevalence of enterococci}

Enterococci were detected in oral rinse samples from one dental student (C1) and 11 endodontic patients (E1-E11). All enterococci were identified as E.faecalis. Details of endodontic patients whose oral rinse samples carried E. faecalis are provided in Table 1. Enterococci were detected in significantly more samples from patients than students $(P=0.0027)$. Viable counts ranged from $1 \times 10$ to $6 \times 10^{3}$ colony forming units per $\mathrm{ml}$ of oral rinse sample.

Oral prevalence of E.faecalis in endodontic patients was not significantly affected by age, gender, smoker status, use of antibiotics within 2 months of sampling, dry mouth medication, symptoms at presentation, and radiographic evidence of periapical lesion associated with the tooth receiving endodontic treatment (Table 2). Data on active caries status, overall oral hygiene assessment and number of dental restorations obtained retrospectively from patient records showed that all patients practiced good oral hygiene and had 10 or more dental restorations. Seven patients had active caries at the time of sampling. The one dental student with oral enterococci was a male non-smoker (age 23 years), who had not taken antibiotics in the previous 2 months and did not take dry mouth medication.

\section{Phenotype}

Details on phenotype and potential virulence traits expressed by oral E. faecalis are presented in Table 3. Four strains produced hemolysin, four strains produced

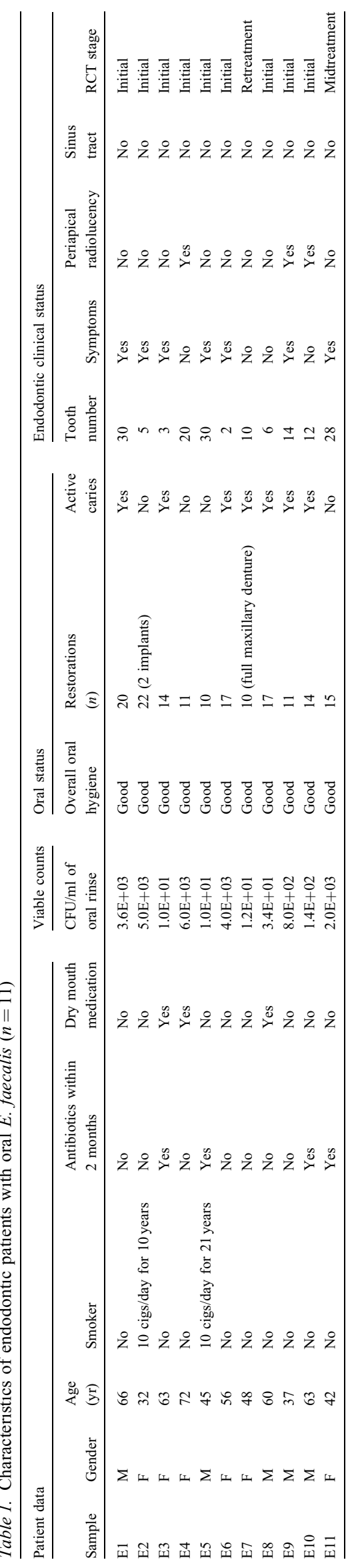

gelatinase and six strains produced bacteriocin. Bacteriocin production was evident only using indicator strains E.faecalis FA2-2, E. faecalis DS16 and E.faecium 409 and not S. aureus ATCC 6538, E. coli DH5 $\alpha$ and E. coli ATCC 29417.

The MIC ranges obtained for antibiotics are shown in Table 4. All strains were susceptible to ampicillin, benzylpenicillin, gentamicin and vancomycin. All strains except one were resistant to clindamycin. Two strains were resistant to erythromycin and one strain to chloramphenicol. Six strains showed intermediate resistance or resistance to tetracycline. The MICs obtained in metal-ion susceptibility tests using oral strains were comparable to those obtained using laboratory strains (Table 5). The strain recovered from a dental student, E. faecalis $\mathrm{C} 1$, was susceptible to all antibiotics except kanamycin and clindamycin, and expressed gelatinase and bacteriocin production and did not exhibit any particular phenotypic characteristics different from strains recovered from endodontic patients (Table 3 ).

E. faecalis E1 was the only strain to respond to pheromone in E. faecalis $\mathrm{JH} 2-2$ culture filtrate. Further analyses using synthetic pheromones showed that E. faecalis E1 responded specifically to the pheromone cPD1 but not to the other pheromones tested (Table 6). This strain produced hemolysin, gelatinase and bacteriocin and was resistant to chloramphenicol, clindamycin, erythromycin, kanamycin and streptomycin, and demonstrated intermediate susceptibility to tetracycline.

\section{Genotype}

PFGE and plasmid evaluation showed that oral E.faecalis exhibited widespread genetic polymorphism (Fig. 1). Smallscale plasmid DNA preparation using an alkaline lysis method showed that seven E.faecalis strains had plasmid DNA (Table 3). Further evaluation using ethidium bromide-CsCl gradient-prepared plasmid DNA from E. faecalis $\mathrm{E} 1$, the oral strain exhibiting the greatest potential virulence activity, indicated that this strain has at least two plasmids (Fig. 2).

\section{Discussion}

The $1 \%$ and $11 \%$ prevalence of oral E. faecalis in oral rinse samples in this crosssectional study is comparable to the $1-5 \%$ previously reported in plaque samples from periodontal patients (22) but considerably lower than the $75 \%$ (cumulative) in multi-site samples collected from eight 
Table 2. Potential factors associated with prevalence of E.faecalis in oral rinse samples from endodontic patients

\begin{tabular}{|c|c|c|c|c|}
\hline & & Present, $n(\%)$ & Absent, $n(\%)$ & $P$ \\
\hline \multicolumn{2}{|l|}{ Number of individuals } & $11(11)$ & $89(89)$ & - \\
\hline \multicolumn{2}{|l|}{ Age (mean) } & 53 years (SD 13 ) & 45 years (SD 15$)$ & n.s. \\
\hline \multirow[t]{2}{*}{ Gender } & Female & $6(55)$ & $36(40)$ & n.s. \\
\hline & Male & $5(45)$ & $53(60)$ & \\
\hline \multirow[t]{2}{*}{ Smoker } & Yes & $2(18)$ & $25(28)$ & \\
\hline & No & $9(82)$ & $64(72)$ & n.s. \\
\hline \multicolumn{5}{|c|}{ Antibiotics within 2 months } \\
\hline & Yes & $4(36)$ & $20(22)$ & n.s. \\
\hline & No & $7(64)$ & $69(78)$ & \\
\hline \multicolumn{5}{|l|}{ Dry mouth medication } \\
\hline & Yes & $2(18)$ & $10(11)$ & n.s. \\
\hline & No & $9(82)$ & $79(89)$ & \\
\hline \multicolumn{5}{|c|}{ Endodontic symptoms at presentation } \\
\hline & Yes & $7(64)$ & $37(42)$ & n.s. \\
\hline & No & $4(36)$ & $52(48)$ & \\
\hline \multicolumn{5}{|c|}{ Endodontic periapical lesion } \\
\hline & Yes & $3(27)$ & $49(55)$ & n.s. \\
\hline & No & $8(73)$ & $40(45)$ & \\
\hline
\end{tabular}

n.s., Not significant $(P>0.05$, Fisher's exact test).

Table 3. Phenotypic characteristics of oral E. faecalis

\begin{tabular}{llllll}
\hline Strain* & Haemolysin & Gelatinase & Bacteriocin & Pheromone response & Plasmid DNA \\
\hline E1 & + & + & + & + & + \\
E2 & & & + & & \\
E3 & & & & & + \\
E4 & & & & + \\
E5 & & & + & + \\
E6 & + & & + & & + \\
E7 & & + & + & + \\
E8 & + & + & & & \\
E9 & + & & & + \\
E10 & & & & & + \\
E11 & & & & & \\
C1 & & & & & + \\
\hline
\end{tabular}

*Oral strains E1-11 from endodontic patients, C1 from dental student.

endodontic patients (14). One reason for this disparity could be the different sampling methods used in each study. The present study used a concentrated oral rinse technique in an attempt to quantitatively evaluate enterococci using one sample of the overall oral cavity (23), in effect combining saliva, soft tissue and dental plaque samples, in contrast to the individual site sampling employed by Gold et al. (14). While the oral rinse method allows a degree of quantification not available using other sampling methods, the precise source of E. faecalis in the oral cavity and the role of these organisms, if any, in dental caries and endodontic infections remains unestablished. Further, it should be noted that cross-sectional studies are limited, being single views of a microflora existing in a complex dynamic environment and not reproducible within the same individual on different occasions $(25,26)$. Future studies could examine the prevalence and characteristics of enterococci recovered in the proximity of dental caries or endodontic infections in longitudinal studies.

E. faecalis was detected in significantly more endodontic patients than dental students with no history of endodontic treatment $(P=0.0027)$. Total numbers of enterococci isolated did not exceed $6 \times 10^{3} / \mathrm{ml}$ of transport medium, in agreement with previous findings (14). Apart from endodontic treatment experience, there were several differences between the two sampling groups, the most significant being smoking habits and age (both $P<0.0001)$, in addition to antibiotic usage $(P<0.0478)$. However, while there were significantly more smokers and antibiotic consumers in the patient group than in the student group, these factors did not appear to influence the presence of E. faecalis in endodontic patients (Table 2). In contrast, increased age has previously been shown to be associated with higher oral prevalence of other enteric microorganisms, for example Enterobacteriaceae (24). To determine if age is a significant factor in the oral prevalence of enterococci, future studies could examine the prevalence in different age groups but would need also to consider other variables such as number and status of restorations, caries incidence, salivary flow rate and periodontal status. The potential role of salivary bacteriophages in the low prevalence of E. faecalis could also be considered in view of a recent

Table 4. Antibiotic susceptibilities (MICs) of oral E. faecalis*

\begin{tabular}{|c|c|c|c|c|c|c|c|c|c|c|c|c|c|c|c|c|c|}
\hline & \multicolumn{3}{|c|}{ NCCLS MIC standards* } & \multicolumn{2}{|c|}{ Laboratory strains } & \multicolumn{12}{|c|}{ Oral strains $^{\dagger}$} \\
\hline & Susc & Inter & Res & JH2-2 & OGI & E1 & E2 & E3 & E4 & E5 & E6 & E7 & E8 & E9 & E10 & E11 & $\mathrm{C} 1$ \\
\hline Ampicillin & $<0.5$ & $1-4$ & $>8$ & 0.75 & 0.75 & 0.38 & 1 & 0.75 & 0.75 & 0.5 & 0.5 & 0.75 & 0.38 & 0.5 & 1 & 0.5 & 0.75 \\
\hline Benzylpenicillin & $<8$ & - & $>16$ & 2 & 3 & 1 & 2 & 2 & 2 & 1.5 & 2 & 1.5 & 1.5 & 1 & 1.5 & 2 & 4 \\
\hline Chloramphenicol & $<4$ & 8 & $>16$ & 3 & 3 & 32 & 4 & 3 & 4 & 1.5 & 3 & 2 & 1.5 & 8 & 4 & 2 & 4 \\
\hline Clindamycin & $<0.5$ & $1-2$ & $>4$ & 8 & 32 & $>256$ & 8 & 24 & 12 & 12 & 16 & 3 & 12 & $>256$ & $>256$ & 8 & 24 \\
\hline Erythromycin & $<0.5$ & $1-4$ & $>8$ & 0.75 & 4 & $>256$ & 3 & 4 & 3 & 1.5 & 3 & 0.094 & 4 & 32 & 2 & 1 & 0.19 \\
\hline Fusidic acid & \multicolumn{3}{|c|}{ No standards available } & $>256$ & 3 & 4 & 3 & 3 & 2 & 3 & 3 & 4 & 1.5 & 3 & 3 & 2 & 3 \\
\hline Gentamicin & $<500$ & - & $>500$ & 16 & 16 & 12 & 8 & 16 & 12 & 12 & 12 & 6 & 12 & 12 & 12 & 16 & 16 \\
\hline Kanamycin & \multicolumn{3}{|c|}{ No standards available } & $>256$ & $>256$ & $>256$ & 48 & $>256$ & 48 & 48 & 48 & 16 & 48 & $>256$ & 128 & 96 & $>256$ \\
\hline Rifampin & \multicolumn{3}{|c|}{ No standards available } & $>256$ & 4 & 0.75 & 4 & 3 & 2 & 3 & 4 & 0.5 & 2 & 1.5 & 2 & 3 & 4 \\
\hline Streptomycin & \multicolumn{3}{|c|}{ No standards available } & 96 & 96 & $>1024$ & 128 & 96 & 96 & 96 & 128 & 64 & 32 & 128 & 128 & 96 & 128 \\
\hline Tetracycline & $<4$ & 8 & $>16$ & 0.19 & 0.19 & 12 & 48 & 0.25 & 16 & 0.64 & 0.094 & 0.094 & 16 & 8 & 16 & 0.064 & 0.19 \\
\hline Vancomycin & $<4$ & $8-16$ & $>32$ & 1.5 & 3 & 3 & 3 & 3 & 1.5 & 1 & 2 & 2 & 1.5 & 3 & 1.5 & 1.5 & 3 \\
\hline
\end{tabular}

"E-test, antibiotic concentrations in $\mu \mathrm{g} / \mathrm{ml}$.

${ }^{\dagger}$ Oral strains E1-11 from endodontic patients, C1 from dental student. 
Table 5. Metal-ion susceptibility (MIC) of oral E.faecalis*

\begin{tabular}{|c|c|c|c|c|c|c|c|c|c|c|c|c|c|c|}
\hline & \multicolumn{2}{|c|}{ Laboratory strains } & \multicolumn{12}{|c|}{ Oral strains ${ }^{\dagger}$} \\
\hline & FA2-2 & OGIRF & E1 & E2 & E3 & E4 & E5 & E6 & E7 & E8 & E9 & E10 & E11 & $\mathrm{C} 1$ \\
\hline $\mathrm{AgNO} 3(\mu \mathrm{M})$ & 78 & 39 & 39 & 78 & 78 & 39 & 78 & 78 & 39 & 39 & 39 & 78 & 39 & 78 \\
\hline $\mathrm{CuSO} 4(\mathrm{mM})$ & 16 & 16 & 31 & 31 & 16 & 8 & 16 & 16 & 16 & 16 & 8 & 16 & 8 & 16 \\
\hline $\mathrm{CdSO} 4(\mathrm{mM})$ & 0.6 & 0.6 & 1.2 & 0.6 & 0.6 & 0.6 & 0.6 & 0.6 & 0.6 & 0.6 & 1.2 & 0.6 & 1.2 & 1.2 \\
\hline $\mathrm{HgCl} 2(\mu \mathrm{M})$ & 16 & 8 & 8 & 16 & 16 & 16 & 16 & 31 & 16 & 16 & 4 & 16 & 8 & 31 \\
\hline ZnSO4 (mM) & 16 & 16 & 8 & 16 & 16 & 8 & 16 & 8 & 16 & 8 & 16 & 8 & 16 & 16 \\
\hline
\end{tabular}

*Broth dilution, MICs recorded as $\mu \mathrm{M}$ or $\mathrm{mM}$ as indicated.

${ }^{\dagger}$ Oral strains E1-11 from endodontic patients, C1 from dental student.

Table 6. Response of E. faecalis E1 to pheromones

\begin{tabular}{|c|c|c|c|c|c|c|c|c|c|c|c|c|c|}
\hline \multirow{2}{*}{$\begin{array}{l}\text { Strains tested for } \\
\text { induced clumping }\end{array}$} & \multirow[b]{2}{*}{ Pheromone } & \multicolumn{11}{|c|}{ Dilution factor $1: 2$} & \multirow{2}{*}{$\begin{array}{l}\text { Negative control } \\
\text { (no pheromone) }\end{array}$} \\
\hline & & 2 & 4 & 8 & 16 & 32 & 64 & 128 & 256 & 512 & 1024 & 2048 & \\
\hline OG1X/pAM373* & cAM373 & + & + & + & + & & & & & & & & - \\
\hline E1 & cAM373 & - & & & & & & & & & & & - \\
\hline OG1SS/pCF10* & $\mathrm{cCF} 10$ & + & + & + & + & + & + & + & + & + & & & - \\
\hline E1 & $\mathrm{cCF} 10$ & - & & & & & & & & & & & - \\
\hline 39-5/pPD1* & cPD1 & + & + & + & + & + & & & & & & & - \\
\hline E1 & cPD1 & + & + & + & + & + & & & & & & & - \\
\hline OG1X/pAD1* & $\mathrm{cAD} 1$ & + & + & + & + & + & + & & & & & & - \\
\hline E1 & $\mathrm{cAD} 1$ & - & & & & & & & & & & & - \\
\hline
\end{tabular}

${ }^{*}$ Known pheromone responsive strains serving as positive controls (5).

E1, oral strain.

report of E. faecalis bacteriophages in $22 \%$ of human saliva samples (1).

Failed endodontically treated teeth have been associated with the presence of intracanal enterococci $(20,28)$ although their role, if any, in the infection process has not been definitively established. Therefore, of particular interest in this study was whether oral enterococci, which could feasibly enter the root canal system due to close proximity, carried potential virulence factors. Four of 11 strains recovered from endodontic patients produced hemolysin, a cytolytic protein capable of lysing erythro- cytes. While hemolysin activity (often plasmid-encoded) has been associated with $60 \%$ of 97 human clinical isolates (17), and virulence in mice (16), the role, if any, of hemolysin production by E. faecalis in the human oral cavity remains unclear.

Four oral strains produced gelatinase. Gelatinases are extracellular zinc endopeptidases capable of hydrolyzing gelatin, collagen and other peptides, and produced by a large proportion of $E$. faecalis isolated from hospitalized patients and patients with endocarditis (7). E. faecalis strains capable of gelatinase production were

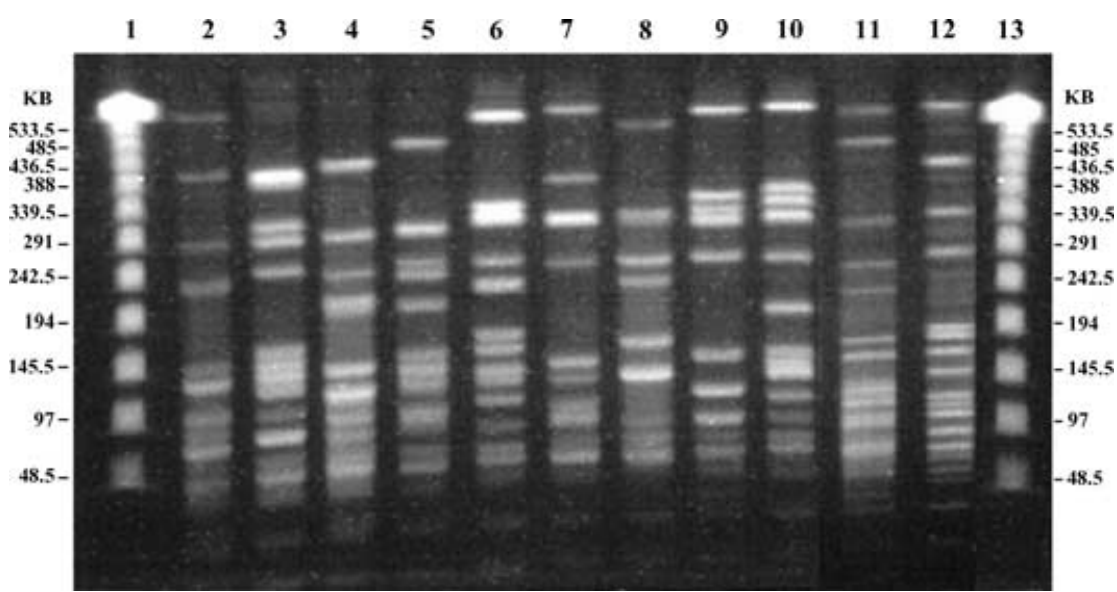

Fig. 1. Pulsed field gel electrophoresis of SmaI-digested genomic DNA from oral E. faecalis strains. Lanes: 1, lambda phage (PFG) DNA standard; 2, E. faecalis E1; 3, E. faecalis E2; 4, E. faecalis E3; 5, E. faecalis $\mathrm{E} 4 ; 6$, E. faecalis E5; 7, E. faecalis $\mathrm{E} 6 ; 8$, E. faecalis $\mathrm{E} 7 ; 9$, E. faecalis $\mathrm{E} 8 ; 10$, E. faecalis E9; 11, E. faecalis E10; 12, E. faecalis E11. shown to contribute to increased severity of endocarditis in animal models (15). Recent data have shown that expression of GelE may contribute to increased dissemination of E.faecalis in high-density environments (32). Previously Gold et al. (14) described E. faecalis $2 \mathrm{SaR}$ (now known as OG1) as a strain recovered from a human oral cavity that exhibited gelatinase activity capable of inducing caries in germ-free rats. In the present study, three strains recovered from endodontic patients produced gelatinase. A retrospective review of patient records showed that these three patients had active caries at the time of sampling. Further analysis of gelatinase activity in oral E.faecalis is ongoing. While oral E. faecalis from another four patients with active caries did not produce gelatinase, future studies could examine the relationship between caries status and gelatinase production by oral enterococci.

The pathogenicity of enterococci has been long established in animal models (19). Further, at the molecular level, it is recognized that E. faecalis participate in plasmid-mediated horizontal transfer of virulence determinants, thus emphasizing the importance of these organisms in nosocomial infections (5). Plasmids are covalently closed circular, double-stranded, supercoiled DNA that can replicate autonomously. They range in size from $\sim 1 \mathrm{~kb}$ to $>200 \mathrm{~kb}$, in contrast to the chromosomal DNA size of $3,218 \mathrm{~kb}$ recently reported for E. faecalis V583 (21). Plasmids frequently 


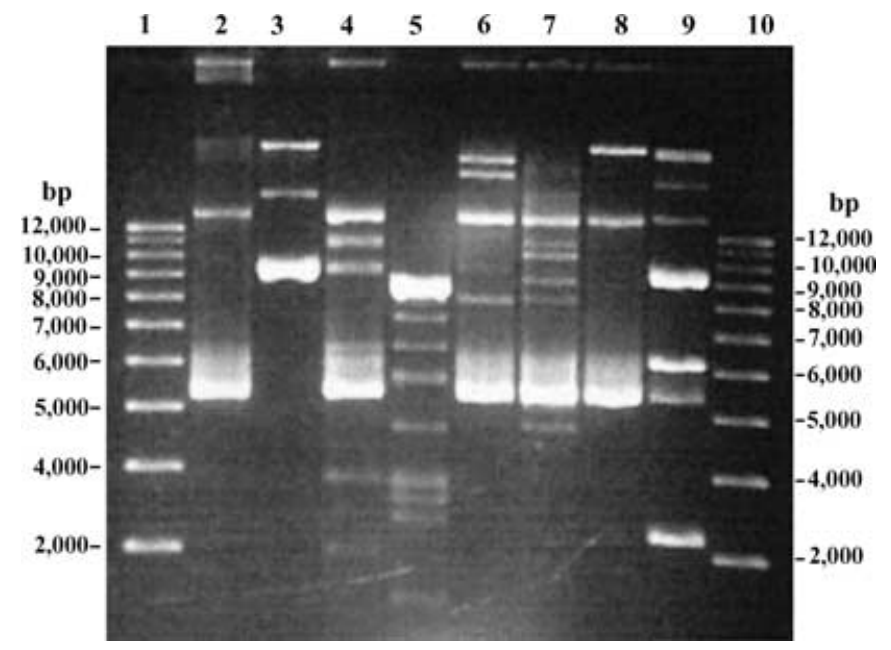

Fig. 2. Agarose gel electrophoresis of $E$. faecalis $\mathrm{E} 1$ plasmid DNA prepared using ethidium bromide$\mathrm{CsCl}$ gradients. Lanes 1 and 10: $1 \mathrm{~Kb}$ Plus ladder. Lane 2. Uncut plasmid DNA; Lanes 3 to 9, overnight digestion with restriction enzymes: Lane 3, BamHI; Lane 4, EcoRI; Lane 5, HinDIII; Lane 6, KpnI; Lane 7, PstI; Lane 8, SmaI; Lane 9, XbaI.

confer traits that facilitate growth/survival under atypical conditions, for example resistance to antibiotics and metal-ions. In the present study, plasmid DNA was recovered from seven oral strains. None of the oral strains demonstrated increased resistance to metal-ions compared to laboratory strains E.faecalis FA2-2 and E.faecalis OG1RF. With the exception of E.faecalis E1, the oral strains with plasmid DNA were generally highly susceptible to the antibiotics studied apart from clindamycin and aminoglycosides, which are associated with intrinsic resistance (29). Two strains exhibited low-level tetracycline resistance $(\mathrm{MIC}=16 \mu \mathrm{g} / \mathrm{ml})$. While plasmid-mediated virulence activity associated with these seven strains remains to be elucidated, E.faecalis E1, which demonstrated hemolysin, gelatinase and bacteriocin production and was resistant to several antibiotics, responded to the pheromone cPD1. Pheromones are small linear peptides secreted by plasmid-free E. faecalis strains. Horizontal transfer of plasmid-encoded virulence determinants can be initiated by pheromones in E. faecalis (5) and some pheromones are chemotactic for human neutrophils (10). E. faecalis E1 clumped in the presence of cPD1, a pheromone known to encode bacteriocin production (34). Bacteriocins are any of a wide variety of protein or peptide antibiotics, commonly encoded by plasmids. In this study, in addition to E. faecalis E1, five other oral strains produced bacteriocin, indicating a capacity for bacteriostatic or bacteriocidal activity directed specifically towards organisms closely related to the bacteriocin-produ- cing strain and not to other genera. Further analysis of E.faecalis E1 plasmid DNA is ongoing.

Pulsed field gel electrophoresis (PFGE) allows separation of DNA fragments produced by rare-cutting restriction enzymes, too large to be resolved using conventional agarose gel electrophoresis. The technique has been utilized in the identification of enterococci (8) and in molecular epidemiological typing of nosocomial enterococci (30). In the present study PFGE of genomic DNA demonstrated the genotypic polymorphism of the oral strains.

In conclusion, enterococci were detected in oral rinse samples from $11 \%$ of 100 patients receiving endodontic treatment and $1 \%$ of 100 dental students with no history of endodontic treatment $(P=0.0027)$. Enterococcal isolates, all identified as E.faecalis, exhibited widespread genetic and phenotypic polymorphism. Potential virulence traits expressed by oral E. faecalis strains included production of hemolysin and gelatinase, and response to pheromones in E. faecalis culture filtrate.

\section{Acknowledgments}

This study was supported by a grant (GM33956) from the National Institute of Health. We thank Susan Donabedian for assistance with pulsed field gel electrophoresis.

\section{References}

1. Bachrach G, Leizerovici-Zigmond M, Zlotkin A, Naor R, Steinberg D. Bacteriophage isolation from human saliva. Lett Appl Microbiol 2003: 36: 50-53.

2. Baker CN, Stocker SA, Culver DH, Thorsberry C. Comparison of the E test to agar dilution, broth dilution and agar diffusion susceptibility testing techniques by using a special challenge set of bacteria. J Clin Microbiol 1991: 29: 533-538.

3. Bender IB, Seltzer S. Combination of antibiotics and fungicides used in treatment of the infected pulpless tooth. J Am Dent Assoc 1952: 45: 293-300.

4. Byström A, Sundqvist G. The antibacterial action of sodium hypochlorite and EDTA in 60 cases of endodontic therapy. Int J Endod 1985: 18: 35-40.

5. Clewell DB, Dunny GM. Conjugation and genetic exchange in enterococci. In: Gilmore MS, ed. The enterococci: pathogenesis, molecular biology and antibiotic resistance. Washington, DC: American Society for Microbiology, 2002: 265-300.

6. Clewell DB, Yagi Y, Dunny GM, Schultz SK. Characterization of three plasmid deoxyribonucleic acid molecules in a strain of Streptococcus faecalis: identification of a plasmid determining erythromycin resistance. J Bacteriol 1974: 117: 283-289.

7. Coque TM, Patterson JE, Steckelberg JM, Murray BE. Incidence of hemolysin, gelatinase, and aggregation substance among enterococci isolated from patients with endocarditis and other infections and from feces of hospitalized and community-based persons. J Infect Dis 1995: 171: 1223-1229.

8. Donabedian S, Chow JW, Shlaes DM, Green M, Zervos MJ. DNA hybridization and contour-clamped homogeneous electric field electrophoresis for identification of enterococci to the species level. J Clin Microbiol 1995: 33: 141-145.

9. Dunny GM, Craig RA, Carron RL, Clewell DB. Plasmid transfer in Streptococcus faecalis: production of multiple sex pheromones by recipients. Plasmid 1979: 2: 454-465.

10. Ember JA, Hugli TE. Characterization of the human neutrophil response to sex pheromones from Streptococcus faecalis. Am J Pathol 1989: 134: 797-805.

11. Engström B. The significance of enterococci in root canal treatment. Odontol Revy 1964: 15: 87-106.

12. Facklam RR, Carvalho MS, Teixeira LM. History, taxonomy, biochemical characteristics, and antibiotic susceptibility testing of enterococci. In: Gilmore MS, ed. The enterococci: pathogenesis, molecular biology and antibiotic resistance. Washington, DC: American Society for Microbiology, 2002: 1-54.

13. Facklam RR, Sahm DF, Teixeira LM. Enterococcus. In: Murray PR, Baron EJ, Pfaller MA, Tenover FC, Yolken RH, eds. Manual of clinical microbiology, 7th edn. Washington, DC: American Society for Microbiology, 1999: 297-305.

14. Gold OG, Jordan HV, van Houte J. The prevalence of enterococci in the human mouth and their pathogenicity in animal models. Arch Oral Biol 1975: 20: 473-477.

15. Gutschik E, Moller S, Christensen N. Experimental endocarditis in rabbits. 3. Significance of the proteolytic capacity of the 
infecting strains of Streptococcus faecalis. Acta Pathol Microbiol Scand [B] 1979: 87: 353-362.

16. Ike Y, Hashimoto H, Clewell DB. Hemolysin of Streptococcus faecalis subspecies zymogenes contributes to virulence in mice. Infect Immun 1984: 45: 528-530.

17. Ike Y, Hashimoto H, Clewell DB. High incidence of hemolysin production by Enterococcus (Streptococcus) faecalis strains associated with human parenteral infections. J Clin Microbiol 1987: 25:1524-1528.

18. Ike Y, Clewell DB, Segarra RA, Gilmore MS. Genetic analysis of the pAD1 hemolysin/ bacteriocin determinant in Enterococcus faecalis: Tn917 insertional mutagenesis and cloning. J Bacteriol 1990: 172: 155-163.

19. MacCallum WG, Hastings TW. A case of acute endocarditis caused by Micrococcus zymogenes (nov. spec.), with a description of the microorganism. J Exp Med 1899: 4: 521-534.

20. Molander A, Reit C, Dahlén G, Kvist T. Microbiological status of root-filled teeth with apical periodontitis. Int Endod J 1998: 31: 1-7.

21. Paulsen IT, Banerjei L, Myers GS, et al. Role of mobile DNA in the evolution of vancomycin-resistant Enterococcus faecalis. Science 2003: 299: 2071-2074.

22. Rams TE, Feik D, Young V, Hammond BF, Slots J. Enterococci in human periodontitis. Oral Microbiol Immunol 1992: 7: 249-252.
23. Samaranayake LP, MacFarlane TW, Lamey P-J, Ferguson MM. A comparison of oral rinse and imprint sampling techniques for the detection of yeast, coliform and Staphylococcus aureus carriage in the oral cavity. $\mathrm{J}$ Oral Pathol Med 1986: 15: 386-388.

24. Sedgley CM, Samaranayake LP. The oral prevalence of aerobic and facultatively anaerobic gram-negative rods and yeasts in Hong Kong Chinese. Arch Oral Biol 1994: 39: 459-466.

25. Sedgley CM, Samaranayake LP, Chan JCY, Wei SHY. A four year longitudinal study of the oral prevalence of enteric gram-negative rods and yeasts in Chinese children. Ora Microbiol Immunol 1997: 12: 183-188.

26. Socransky SS, Manganiello SD. The oral microbiota of man from birth to senility. J Periodontol 1971: 42: 485-496.

27. Sundqvist G. Associations between microbial species in dental root canal infections. Oral Microbiol Immunol 1992: 7: 257-262.

28. Sundqvist G, Figdor D, Persson S, Sjögren U. Microbiologic analysis of teeth with failed endodontic treatment and the outcome of conservative re-treatment. Oral Surg Oral Med Oral Pathol Oral Radiol Endod 1998: 85: 86-93

29. Tannock GW, Cook G. Enterococci as members of the intestinal microflora of humans. In: Gilmore MS, ed. The enterococci: pathogenesis, molecular biology and antibiotic resistance. Washington, DC:
American Society for Microbiology, 2002: 101-132.

30. Thal L, Donabedian S, Robinson-Dunn B, et al. Molecular analysis of glycopeptideresistant Enterococcus faecium isolates collected from Michigan hospitals over a 6 year period. J Clin Microbiol 1998: 36 3303-3308.

31. Walsh TJ, Venanzi WE, Dixon DM. Quantification of medically important Candida species and Torulopsis glabrata by a spiral inoculation system : correlation with pour plate and spread plate methods. J Clin Microbiol 1985: 22: 745-747.

32. Waters CM, Antiporta MH, Murray BE, Dunny GM. Role of the Enterococcus faecalis GelE protease in determination of cellular chain length, supernatant pheromone levels, and degradation of fibrin and misfolded surface proteins. J Bacteriol 2003: 185: 3613-3623.

33. Weaver KE, Clewell DB. Regulation of the pAD1 sex pheromone response in Enterococcus faecalis: construction and characterization of lacZ transcriptional fusions in a key control region of the plasmid. J Bacteriol 1988: 170: 4343-4352.

34. Yagi Y, Kessler RE, Shaw JH, Lopatin DE, An F, Clewell DB. Plasmid content of Streptococcus faecalis strain 39-5 and identification of a pheromone (cPD1)-induced surface antigen. J Gen Microbiol 1983: 129 $1207-1215$. 